\title{
Effects of Chronic Ethanol Intake on Mobilization and Excretion of Cholesterol in Baboons
}

\author{
Claude Karsenty, Enrique Baraona, Markku J. Savolainen, and Charles S. Lieber \\ Alcohol Research and Treatment Center and Section of Liver Disease and Nutrition, Bronx Veterans Administration \\ Medical Center and Mount Sinai School of Medicine, City University of New York, New York 10468
}

\begin{abstract}
To investigate the effects of chronic ethanol administration on the mobilization and excretion of cholesterol, turnover and balance studies were carried out in baboons pair-fed cholesterolfree diets containing $50 \%$ of energy either as ethanol or as additional carbohydrate for several years. Ethanol feeding increased free cholesterol in all plasma lipoprotein fractions, and esterified cholesterol in very low density lipoprotein, intermediate density lipoprotein, and high density lipoprotein (HDL). The major increase occurred in HDL, mainly as esterified cholesterol. The latter was associated with decreased transfer of esterified cholesterol from HDL to low density lipoprotein. By contrast, the smaller increase in HDL-free cholesterol was associated with increased turnover in the plasma, increased splanchnic uptake, and increased fecal excretion of plasma cholesterol, mainly as neutral steroids. Cholesterol extraction predominated over release in the splanchnic vascular bed, suggesting that the excess of cholesterol excreted in the feces originated in extrasplanchnic tissues. Thus, these findings indicate that alcohol consumption favors mobilization of tissue free cholesterol for hepatic removal and excretion. By contrast the increase in HDL-cholesterol (mainly esterified) appears to be a poor indicator of cholesterol mobilization.
\end{abstract}

\section{Introduction}

Epidemiologic studies suggest that moderate alcohol consumption decreases the risk of coronary heart disease (1). In nonhuman primates (Macaca nemestrina), chronic feeding of ethanol (36\% of total energy) either with a low or a high cholesterol diet, decreased aortic and coronary atherosclerosis (2). The mechanism for this effect remains unknown. In vivo $(3,4)$ and in vitro $(5,6)$ studies indicate that high density lipoproteins (HDL) promote cholesterol efflux from the cells. Therefore, the observation that alcohol increases plasma concentrations of $\operatorname{HDL}(7,8)$, particularly the cholesterol moiety (HDL-C) ${ }^{1}$ (9), suggested that it is via this lipoprotein fraction that ethanol enhances mobilization of tissue cholesterol for hepatic disposition into bile. However, the possibility that the increased HDL-C could be due to defective removal of choles-

Received for publication 19 May 1983 and in revised form 11 January 1984.

1. Abbreviations used in this paper: EC, esterified cholesterol; FC, free cholesterol; HDL-C, cholesterol moiety of high density lipoprotein; LCAT, lecithin cholesterol acyltransferase.

The Journal of Clinical Investigation, Inc.

Volume 75, March 1985, 976-986 terol from the blood rather than to increased mobilization from tissue has not been ruled out. This study was undertaken to investigate whether chronic ethanol administration (under nutritionally controlled conditions and in the absence of severe liver injury) does indeed promote net losses of cholesterol from the body and to assess sources and pathways of disposition for this cholesterol. To this effect, we chose the baboon as an experimental model because of its close resemblance to humans with regard to lipoprotein composition (10), cholesterol metabolism (11), and its ability to develop atherosclerosis (12) and a spectrum of alcoholic liver injury (13).

\section{Methods}

Materials. $\left[4-{ }^{14} \mathrm{C}\right]$ Cholesterol $(60 \mathrm{mCi} / \mathrm{mmol}),\left[1,2-{ }^{3} \mathrm{H}\right]$ cholesterol $(40.7$ $\mathrm{Ci} / \mathrm{mmol})$, and $\left[\right.$ carboxyl $\left.-{ }^{14} \mathrm{C}\right]$ cholic acid $(52 \mathrm{mCi} / \mathrm{mmol})$ were purchased from New England Nuclear (Boston, MA). The composition of the liquid diets was as previously reported (13). They contain $18 \%$ of total energy as protein, $21 \%$ as fat, $11 \%$ as carbohydrate, $50 \%$ either as ethanol or as additional carbohydrate, and no cholesterol. The liquid diet contains $1 \%$ fiber, which is equivalent to $5 \%$ in solid diets.

Animal procedures. 12 male and 16 female baboons (Papio hamadryas) of an estimated age of 3-5 yr were pair-fed the liquid diets described above for $1-9 \mathrm{yr}$ (mean $=27 \mathrm{mo})$. The daily consumption of the liquid diets was $66 \pm 5 \mathrm{ml} / \mathrm{kg}$ of body weight, representing an ethanol intake of $4.6 \pm 0.3 \mathrm{~g} / \mathrm{kg}$ per day. The pair-fed controls gained weight ( $100 \mathrm{~g}$ per month), whereas the body weight of the alcohol-fed animals remained constant $(12.0 \pm 0.4 \mathrm{~kg}$ initially vs. $11.3 \pm 0.5$ at the end of this study; NS) despite isocaloric feeding. For the duration of the turnover and balance studies reported here, there were no detectable changes in body weight. Alcohol-fed animals consumed their diets evenly during the 24-h period; blood ethanol concentrations (14), measured at the middle of the feeding period, were $38 \pm 5 \mathrm{mM}$. By contrast, the controls ingested their rations during the few hours after the servings. To achieve a similar degree of fasting, the diet of the alcohol-fed baboons was removed $18 \mathrm{~h}$ before starting the procedures indicated below, whereas in the controls, the removal of the diet was preceded by administration of $10 \mathrm{ml}$ of control diet per kilogram body weight for $4 \mathrm{~h}$. After the fasting period, no ethanol was detected in the blood. The animals were then anesthetized with ketamine- $\mathrm{HCl}(10$ $\mathrm{mg} / \mathrm{kg}$ of body weight) and restrained, and venous and arterial blood were collected from the femoral vessels. In four pairs, fasting cholesterol levels were compared with those after intragastric administration of 10 $\mathrm{ml}$ of the corresponding diet per kilogram body weight.

The animals were subjected to either percutaneous needle or surgical liver biopsies at intervals of $\sim 6 \mathrm{mo}$; thus, every animal had a histologic assessment of the liver lesions within 2-3 mo of any experiment. At the time of laparotomy, simultaneous blood samples were obtained from the portal vein and from the femoral artery to assess for net extraction or release of cholesterol in the splanchnic (nonhepatic) vascular bed. The liver volume was estimated roentgenologically (15).

Analysis of the liver samples. Specific gravity was assessed from the displacement of saline by a weighed piece of liver. Liver lipids were extracted (16) and fractionated by thin layer chromatography (17). Triglycerides were eluted and measured by determination of the ester 
bonds (18). Liver free and total cholesterol were measured colorimetrically $(19,20)$.

Analysis of the serum samples. The cholesterol concentration in the various lipoprotein fractions (isolated as described below) was assessed with cholesterol oxidase, either by measuring the oxygen consumption in a model 2 cholesterol analyzer (Beckman Instruments, Inc., Palo Alto, CA) or by measuring $\mathrm{H}_{2} \mathrm{O}_{2}$ generation (21). The methods were validated against each other at high concentrations, and with the $\sigma$-phthalaldehyde method (22) at low concentrations. Total and free cholesterol were determined separately either in the presence or in the absence of cholesteryl ester hydrolase, respectively, and the esterified fraction was calculated from the difference. The coefficient of variation was $<5 \%$. Fractionation of the serum into various lipoprotein classes resulted in $\sim 90 \%$ recovery of the cholesterol. Serum triglyceride concentrations were measured enzymatically (23) using triglyceride ultraviolet assay reagents (Beckman Instruments, Inc.). Lecithin cholesterol acyltransferase (L-CAT) activity was measured according to Stokke and Norum (24).

Blood was collected from the fasting baboons in tubes containing $1 \mathrm{mg}$ of EDTA tetrasodium salt, per milliliter of blood, $2 \mathrm{~d}$ before the initiation of the turnover studies. The plasma was immediately separated by centrifugation at $2,000 \mathrm{rpm}$ at $4^{\circ} \mathrm{C}$ for $20 \mathrm{~min}$. The $\mathrm{HDL}$ were doubly labeled in free cholesterol and esterified cholesterol (25) in order to assess the esterification of free cholesterol taking place in vivo during the turnover studies. $7 \mathrm{ml}$ of plasma was incubated at $37^{\circ} \mathrm{C}$ for $4 \mathrm{~h}$ with $5-\mathrm{mm}$ disks of filter paper that had been impregnated with $80 \mu \mathrm{Ci}$ of $\left[{ }^{3} \mathrm{H}\right]$ cholesterol. The filter papers were changed twice at 80 min intervals. This incubation promotes physical exchange of free cholesterol and in vitro esterification by L-CAT activity present in the plasma. A second 4-h incubation with disks impregnated with $10 \mu \mathrm{Ci}$ of $\left[{ }^{14} \mathrm{C}\right]$ cholesterol at $4^{\circ} \mathrm{C}$ (to inhibit L-CAT activity) labeled only free cholesterol with ${ }^{14} \mathrm{C}$.

Lipoproteins were then fractionated by a single 45 -min centrifugation at $360,000 \mathrm{~g}$ at $10^{\circ} \mathrm{C}$ in a vertical rotor (VTi 65) in an ultracentrifuge (L8-80; Beckman) (26). The density of $1.5 \mathrm{ml}$ of plasma was adjusted to $1.300 \mathrm{~g} / \mathrm{ml}$ by addition of $\mathrm{NaCl}$ and $\mathrm{KBr}$, the sample was transferred into $5 \mathrm{ml}$ of Beckman Quick-Seal tubes and overlayered with 1.006 saline solution containing $0.01 \%$ EDTA. The separation of very low density lipoprotein (VLDL) $(d<1.006)$, intermediate density lipoprotein (IDL) (d 1.006-1.019), low density lipoprotein (LDL) (d 1.019-1.063), and HDL ( $d$ 1.063-1.210), was followed by staining of the plasma with activated Fat Red 7B (27). The fractions were collected in a Beckman Fraction Recovery System, pushing the contents from below with a 1.346 density $\mathrm{KBr}$ solution. The HDL fractions were pooled, dialyzed overnight against $0.9 \% \mathrm{NaCl}$ and $0.01 \%$ EDTA (pH 7.4), and passed through a $0.22 \mu \mathrm{m}$ sterile filter (Millipore Corp., Bedford, MA) before injection in to the baboons. The labeled HDL migrated in agarose gel electrophoresis (28) as a single band, identical to the native HDL. On recentrifugation, $88.2 \%$ of the radioactivity was recovered in the density range of HDL. 59\% of the ${ }^{3} \mathrm{H}$-radioactivity was in free cholesterol and $41 \%$ in esterified cholesterol, whereas $100 \%$ of the ${ }^{14} \mathrm{C}$ radioactivity was in free cholesterol. No cholesterol was detected in the $d>1.21$ fraction.

$H D L$-cholesterol turnover. Five pairs of fasted baboons were injected intravenously with the autologous labeled HDL. Venous blood samples were collected in ice-cold EDTA-containing tubes at frequent intervals (as indicated in Figs. 1-4) for $31 \mathrm{~h}$. Plasma separation at $4^{\circ} \mathrm{C}$ and lipoprotein fractionation at $10^{\circ} \mathrm{C}$ were carried out within $1-2 \mathrm{~h}$. To test for the extent of ex vivo transfer/exchange of free cholesterol among lipoproteins, we mixed HDL labeled with $\left[{ }^{14} \mathrm{C}\right]$ free cholesterol with autologous blood and kept the specimens at $4^{\circ} \mathrm{C}$ for 1 and $2 \mathrm{~h}$ before starting the 45-min lipoprotein ultracentrifugation. We found that $12 \%$ and $16 \%$ of the free cholesterol radioactivity was recovered in fractions other than HDL, respectively. The short centrifugation times used in this study greatly reduced ex vivo exchange of free cholesterol among the lipoproteins and probably minimized the alterations of HDL induced by classical ultracentrifugation (29). Lipids were extracted from the lipoprotein fractions (16). Free and esterified cholesterol were separated by thin-layer chromatography and stained with iodine vapors. After scratching the bands, free and esterified cholesterol bands were eluted and used for measuring the concentration (22), and for liquid scintillation counting in $0.55 \% 2,5$-diphenyloxazole$0.22 \%$ 1,4-bis[2-(5-phenyloxazolyl)]benzene-containing toluene.

Because the injected HDL was labeled with ${ }^{3} \mathrm{H}$ in both the esterified and the free cholesterol, the fraction derived from in vivo esterification of $\left[{ }^{3} \mathrm{H}\right]$ free cholesterol was subtracted from the total ${ }^{3} \mathrm{H}$-radioactivity in HDL-esterified cholesterol, in order to assess the clearance of the injected HDL-esterified cholesterol. To this effect, we used the ${ }^{14} \mathrm{C}$ label, which initially was present only in HDL-free cholesterol but which appeared in the esterified fraction during the course of the experiment. For each interval, the amount of free cholesterol being esterified was calculated by dividing the ${ }^{14} \mathrm{C}$-radioactivity in HDLesterified cholesterol by the mean specific activity of $\left[{ }^{14} \mathrm{C}\right] \mathrm{HDL}$-free cholesterol during that interval. The latter multiplied by the mean specific activity of $\left[{ }^{3} \mathrm{H}\right]$ free cholesterol, provides the fraction of ${ }^{3} \mathrm{H}$ radioactivity due to in vivo esterification of HDL-free cholesterol. Kinetic parameters were calculated from semilogarithmic plots of the specific activities versus time, using a two-pool model (30) (see Appendix). A similar approach has been applied to the analysis of the kinetics of total body cholesterol $(31,32)$. However, in the latter studies, the sampling was initiated $8 \mathrm{~h}$ or more after the administration of the labeled cholesterol or its precursors (when the cholesterol specific activity in lipoproteins and elsewhere had become similar) and lasted for several weeks; under these conditions, plasma cholesterol is only a small fraction of the rapidly exchangeable pool. By contrast, we focused on the more rapidly exchangeable pools of both plasma free and esterified cholesterol because their changes are likely to have a greater impact in determining plasma cholesterol concentrations than the slower turning over pools assessed in the long-term studies. This approach was made possible by rapid lipoprotein fractionation, which minimizes the ex vivo transfer/exchange of cholesterol (33). $31 \mathrm{~h}$ after the injection of the labeled HDL-C, the specific activity had decreased to $<10 \%$ of the initial $(2-\mathrm{min})$ values. In some of the pairs, the HDL-C specific activity was measured at later times $(7,42,120,140$, or $180 \mathrm{~d})$. These values decreased with time from $3-5 \%$ of the initial specific activity to $0.08-0.09 \%$, defining a third, much slower exponential decay with a half-life of $\sim 30 \mathrm{~d}$, which is similar to that previously reported for this slow component in the baboon (32). At these times, no consistent differences were noted between the HDL-C specific activities of alcohol-fed and control baboons.

Net splanchnic removal or production of cholesterol. In six pairs, a polyurethane catheter (cobra type I, size 7F; USCI Cardiology \& Radiology Products, Billerica, MA) was introduced percutaneously into the femoral vein and advanced into the hepatic vein under fluoroscopy. Another cannula was introduced into the femoral artery and placed in the aorta. Samples for the estimation of the hepatic plasma flow were taken every $15 \mathrm{~min}$ for $5 \mathrm{~h}$, and the flow was calculated from the plasma volume measured with Evans blue dye (34) and the continuous extraction of indocyanine green (CardioGreen; Hanson, Westcott \& Dunning, Inc., Baltimore, MD) (35). After 1-2 $\mathrm{h}$ of recovery from anesthesia, several simultaneous blood samples were collected from the aorta and the hepatic vein of the restrained animals for cholesterol measurements over a 3-4-h period. Some animals required additional small doses $(1-2 \mathrm{mg} / \mathrm{kg}$ ) of ketamine. Blood flow and cholesterol extractions were similar at various times after administration of the drug and these values were therefore averaged. The product of the arterio-hepatic venous differences in plasma cholesterol and the plasma flow was calculated to assess net extraction from, or release into, the blood.

Steroid balance. The fecal excretion of steroids was measured by the isotopic balance method (36), as applied to the baboon (11). This technique measures fecal steroids of plasma origin. The feces were collected for 6 consecutive days $6 \mathrm{wk}$ or more after the injection of the labeled autologous HDL, at a time when isotopic equilibrium 
between plasma cholesterol and biliary cholesterol and bile acids had been achieved (11). Slanted stainless steel trays below the cages allowed for drainage of the urine into a collecting beaker while retaining the feces. Chromic oxide $(150 \mathrm{mg}$ ) was given daily in the liquid diets for $5 \mathrm{~d}$ before and throughout the fecal collection period to correct for steroid losses and variations in fecal flow (37). Previous studies in baboons (Mott, G. E., personal communication) indicated no significant intestinal degradation of neutral steroids, making it unnecessary to use $\beta$-sitosterol to control for this factor. The initiation and termination of the fecal collection period were marked by adding $150 \mathrm{mg}$ of carmine red to the diet. Plasma cholesterol specific activity was measured at the beginning and at the end of the fecal collection period.

The feces were homogenized in 1 vol of water. Neutral fecal steroids were extracted in petroleum ether after mild saponification (38). Bile acids were extracted after alkaline hydrolysis of the bile salt conjugates (39). $\left[4-{ }^{14} \mathrm{C}\right] \mathrm{Cholesterol}$ and $\left[\right.$ carboxyl $\left.-{ }^{14} \mathrm{C}\right]$ cholic acid were used to assess the degree of recovery. The extracts were dried under nitrogen, partially decolorized overnight with $200 \mu \mathrm{l}$ of hydrogen peroxide, and counted in a chemiluminescence-inhibiting scintillation solution (Dimiscint; National Diagnostics, Inc., Somerville, NJ), using ${ }^{3} \mathrm{H}$ and ${ }^{14} \mathrm{C}$ internal standards for quench correction.

The amount of fecal endogenous neutral steroids was calculated by dividing the radioactivity of the extracts by the specific activity of plasma cholesterol existing $2 \mathrm{~d}$ before the midpoint of the fecal collection period. The transit time was the same in alcohol-fed and control baboons as judged by the appearance of the carmine coloration of the feces. In the case of acidic steroids, the plasma specific activity $4 \mathrm{~d}$ before the midpoint of the collection period was chosen to correct for the transit time and for the effects of the entero-hepatic recirculation of bile acids (11).

Statistics. Values are expressed by their means \pm the standard error of the mean (SEM). Yalues obtained in the alcohol-fed animals were compared with those obtained in their pair-fed controls and the mean of the individual differences was tested by the Student's $t$ test (paired comparisons) (40). The paired $t$ test was also applied to comparisons between simultaneous samples in the same animal. In comparisons between alcohol-fed animals with various degrees of liver injury (group comparisons), the $t$ test was applied to the difference of the group means. Changes over time occurring in the same animal were tested by one-way analysis of the variance. The regression lines were obtained using the least-square technique applied to exponential trends. The significance of the differences between regressions was assessed by analysis of the co-variance applied to either the comparisons of slopes or the elevations in the case of parallel lines $(40)$.

\section{Results}

Effects of ethanol feeding on liver chemistry and morphology. As previously reported (13), the alcohol-fed baboons developed various stages of alcoholic liver disease. All alcohol-fed baboons had some degree of fat accumulation, ballooning of the hepatocytes, and ultrastructural alterations, but no signs of cholestasis. Except for one alcohol-fed baboon with mild hyperbilirubinemia $(1.9 \mathrm{mg} / \mathrm{dl})$ all others were anicteric $(0.25 \pm 0.05$ $\mathrm{mg} / \mathrm{dl}$ vs. $0.23 \pm 0.06 \mathrm{mg} / \mathrm{dl}$ in controls). The animals could be divided into four groups on the basis of the degree of associated fibrosis, namely, ( $a$ ) no fibrosis, $(b)$ deposition of fibrous tissue around the terminal hepatic venules (perivenular fibrosis), $(c)$ development of fibrous septa spreading from this area into the lobules, and $(d)$ complete distortion of the normal structure by septa with the formation of nodules (cirrhosis).

In the ethanol-fed baboons there was enlargement of the liver $(30.6 \pm 1.6 \mathrm{~g} / \mathrm{kg}$ body weight vs. $24.8 \pm 1.0 \mathrm{~g} / \mathrm{kg}$ in the controls; $n=14$ pairs; $P<0.001$ ). Also, there was a marked increase in liver triacylglycerol concentrations $(136 \pm 15 \mathrm{mg} / \mathrm{g}$ of liver vs. $25 \pm 5 \mathrm{mg} / \mathrm{g}$ in the controls; $n=14$ pairs, $P<0.001)$. By contrast, there were no significant changes in the concentration of cholesterol, either the free $(1.18 \pm 0.13$ $\mathrm{mg} / \mathrm{g}$ vs. $1.19 \pm 0.17 \mathrm{mg} / \mathrm{g}$ in the controls; $n=14$ pairs; NS) or the esterified form $(0.88 \pm 12 \mathrm{mg} / \mathrm{g}$ vs. $0.94 \pm 0.17 \mathrm{mg} / \mathrm{g}$ in the controls; $n=14$ pairs; NS). There was also no difference in hepatic cholesterol content at various stages of alcoholic liver injury.

Effect of ethanol feeding on plasma cholesterol. Significant hypercholesterolemia was found only at early stages of alcoholic liver injury (fatty liver with or without perivenular fibrosis). Thus, all the following studies concerning the mechanism of the hypercholesterolemia were carried out at these early stages of liver injury. The concentrations of free and esterified cholesterol in different lipoprotein fractions are presented in Table I. In every fraction, free cholesterol was significantly

Table I. Effects of Chronic Ethanol Feeding on Fasting Plasma Triacylglycerols and Cholesterol in Baboons*

\begin{tabular}{llllc}
\hline & VLDL & IDL & LDL & HDL \\
\hline Triacylglycerols & $d<1.006$ & $d 1.006-1.019$ & $d 1.019-1.063$ & $d 1.063-1.210$ \\
Ethanol-fed baboons & $389 \pm 120$ & $176 \pm 49$ & $271 \pm 39$ & $427 \pm 30$ \\
Pair-fed controls & $63 \pm 10$ & $109 \pm 14$ & $130 \pm 19$ & $240 \pm 40$ \\
FC & $P<0.01$ & $P<0.01$ & $P<0.01$ & $P<0.01$ \\
Ethanol-fed baboons & $81 \pm 10$ & & & $323 \pm 20$ \\
Pair-fed controls & $26 \pm 4$ & $121 \pm 10$ & $220 \pm 18$ & $285 \pm 21$ \\
EC & $P<0.001$ & $P<0.005$ & $P<0.005$ & $P<0.05$ \\
Ethanol-fed baboons & $111 \pm 15$ & & & \\
Pair-fed controls & $41 \pm 10$ & $221 \pm 31$ & $851 \pm 70$ & $1285 \pm 145$ \\
EC/FC ratio & $P<0.001$ & $P<0.01$ & NS & $915 \pm 70$ \\
Ethanol-fed baboons & $1.50 \pm 0.17$ & $1.79 \pm 0.14$ & & $P<0.025$ \\
Pair-fed controls & $1.48 \pm 0.25$ & $1.43 \pm 0.11$ & $2.61 \pm 0.13$ & $3.42 \pm 0.16$ \\
& NS & NS & $3.46 \pm 0.44$ & $3.19 \pm 0.11$
\end{tabular}

* Mean $\pm \mathrm{SE}$ ( $\mu \mathrm{mol} /$ liter of plasma) for 14 baboons pair-fed $50 \%$ of energy either as ethanol or as additional carbohydrate. Statistical significance was calculated using Student's $t$ test for paired comparisons. 
higher in the ethanol-fed animals than in the pair-fed controls. Esterified cholesterol was significantly increased in the VLDL, IDL, and HDL fractions of the ethanol-fed baboons, with no significant changes in LDL. This resulted in a significant decrease of the esterified/free cholesterol ratio in LDL, whereas this ratio tended to be increased (although not significantly) in all other fractions. The greatest proportional increase in cholesterol occurred in the $d<1.019$ lipoproteins (VLDL and IDL). In absolute terms, however, most of the increase occurred as cholesteryl esters in HDL.

There was no difference in cholesterol concentration between arterial and femoral venous samples either in alcoholfed baboons or in the pair-fed controls. In four pairs, $10 \mathrm{ml}$ of the corresponding diet per $\mathrm{kg}$ body weight were administered intragastrically to compare fasting and postprandial cholesterol levels. A small and progressive reduction in plasma cholesterol concentration was noted in the alcohol-fed animals with an $18 \%$ decrease $(P<0.01) 4 \mathrm{~h}$ after feeding. No significant changes occurred in the controls. The decrease in the alcoholfed animals was associated with a postprandial rise in serum triglycerides (from $970 \pm 296$ to $1,403 \pm 387 \mu \mathrm{mol} /$ liter after 4 $\mathrm{h} ; P<0.01$ ) whereas no significant changes occurred in the controls ( $548 \pm 38$ vs. $498 \pm 69 \mu \mathrm{mol} / \mathrm{liter}$; NS).

$H D L$-free cholesterol (FC) turnover. The mean decay curves of specific activities of HDL-FC in the alcohol-fed and control groups are shown in Fig. 1, and the kinetic parameters derived from the two-pool analysis of the individual curves are given in the Appendix. The specific activity of HDL-FC decayed very rapidly during the first $5 \mathrm{~h}$ and more slowly thereafter. The specific activity of HDL-FC remained two- to fourfold higher than that of LDL-FC for the first $4 \mathrm{~h}$ after injection of labeled HDL-FC. Thereafter, the specific activity of FC in LDL was not significantly different from that in HDL.

Ethanol feeding produced no significant changes in the half-lives of either the rapid or the slow phases of the decay of free cholesterol specific activity or in the rate constants for removal or transfer between the pools (Appendix). However, the total amount of free cholesterol being turned over (total or absolute catabolic rate) was significantly increased, mainly as a reflection of the higher plasma cholesterol concentrations and the larger pool size in the ethanol-fed baboons. In the latter, the production rate of new free cholesterol incorporated into HDL (excluding the recycled one) was more than twice that of control baboons. The kinetics of the rapidly exchangeable pool of HDL-FC most likely reflect both exchange/transfer of free cholesterol between plasma HDL and other lipoproteins and some cell membranes, and esterification to HDL-esterified cholesterol (EC). The size of this pool represented $86 \%$ and $84 \%$ of the plasma HDL-FC in alcohol-fed and control baboons, respectively.

Esterification of HDL-FC. During the course of the turnover studies, there was the appearance of ${ }^{14} \mathrm{C}$, injected as free cholesterol, into the esterified fraction of the HDL. The rate of cholesterol esterification was significantly greater in the alcohol-fed than in the control baboons $(44.1 \pm 5.2 \mathrm{nmol}$ of cholesterol/milliliter of plasma per hour vs. $21.6 \pm 4.7 \mathrm{nmol} /$ $\mathrm{ml}$ per hour in controls; $P<0.01$ ) (Fig. 2). Also, in vitro (24) the amount of cholesterol being esterified was significantly greater in the alcohol-fed animals $(54.0 \pm 4.8 \mathrm{nmol} / \mathrm{ml}$ per hour vs. $37.5 \pm 3.9 \mathrm{nmol} / \mathrm{ml}$ per hour in controls; $n=5$ pairs;

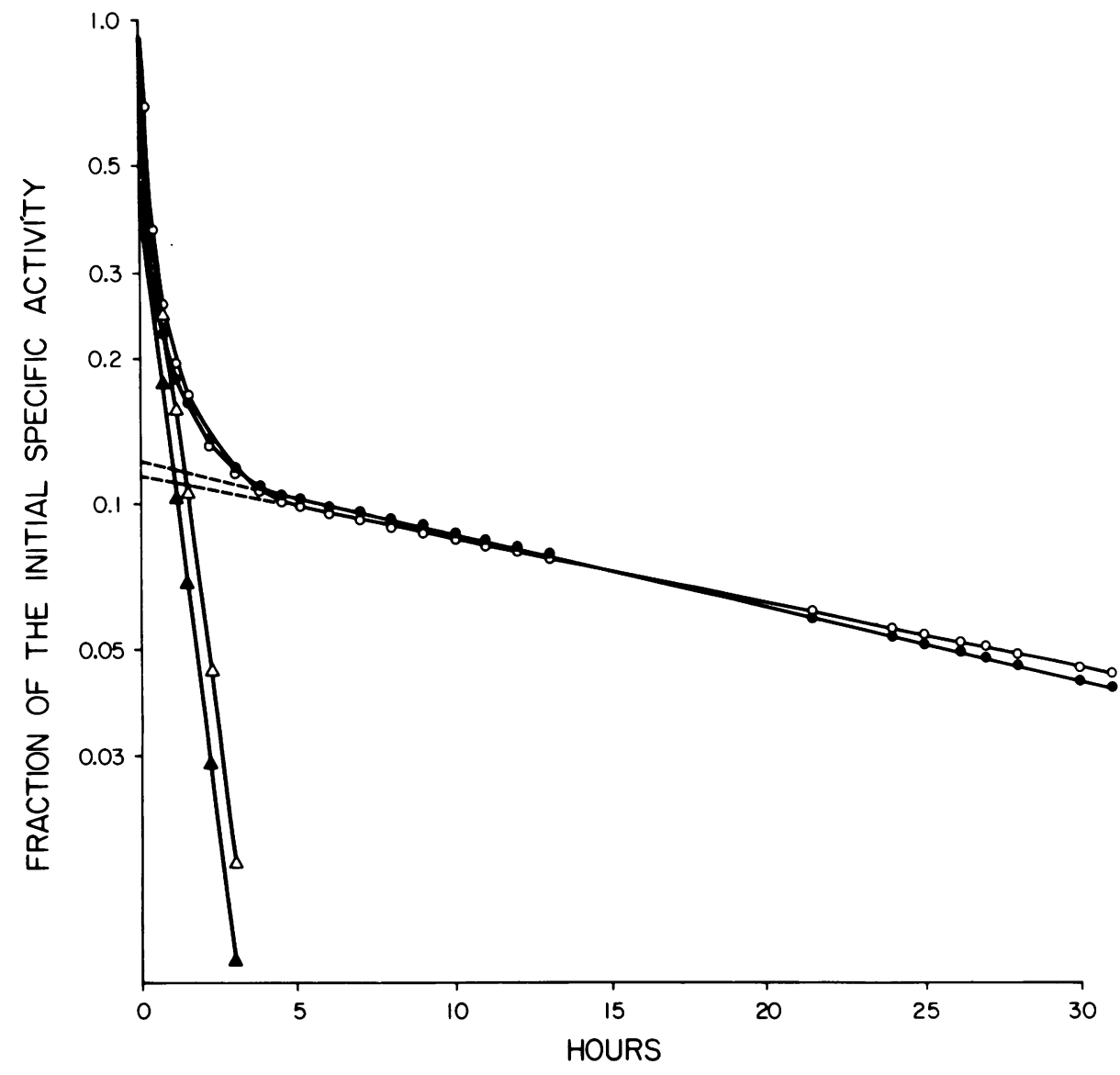

Figure 1. Average decay curve of free cholesterol specific activity in plasma HDL of five pairs of baboons chronically pair-fed $50 \%$ of energy either as ethanol or as additional carbohydrate. To average the individual curves, the specific activities were expressed as a fraction of the values in the 2-min plasma samples. The decay followed two-pool kinetics. Experimental values were obtained at each of the indicated circles. Values for the first exponential are illustrated by triangles. They were calculated by subtracting from the experimental data the extrapolated value of the second exponential. There were no significant differences in half-lives of either exponential between alcohol-fed and control baboons. Ethanol-fed baboons: •, experimental values; $\Delta$, calculated values. Pair-fed controls: $O$, experimental values; $\Delta$, calculated values. 


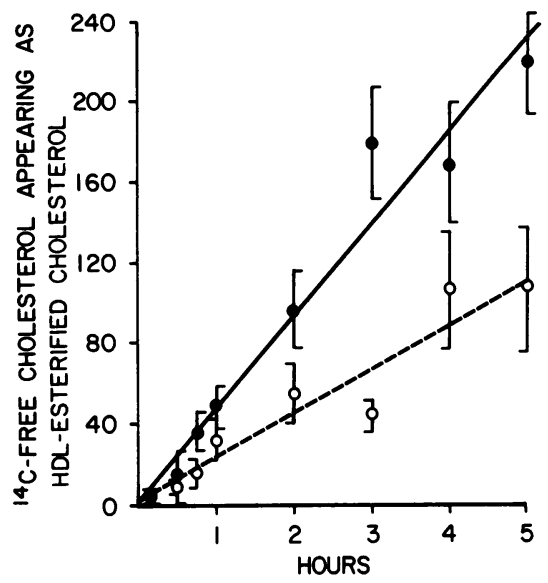

Figure 2. Rate of esterification of $\left[{ }^{14} \mathrm{C}\right]$ free cholesterol in HDL (nanomoles per milliliters of plasma). Baboons pair-fed either ethanol or isocaloric carbohydrate were injected with their own HDL labeled in vitro with ${ }^{14} \mathrm{C}$ in free cholesterol. The amount esterified was calculated from the ${ }^{14} \mathrm{C}$-incorporation in the esterified fraction and the specific activity of $\left[{ }^{14} \mathrm{C}\right]$ free cholesterol. There was more rapid esterification of HDL-FC in alcohol-fed baboons than in the controls $(P<0.01)$. •, ethanol-fed baboons; O, pair-fed controls.

$P<0.02$ ) (if we assume complete equilibration between the labeled free cholesterol added with the free cholesterol present in the plasma). However, the fractional conversion rate was similar $(6.03 \pm 0.53 \%$ vs. $6.37 \pm 0.67 \%$, in controls; NS). These in vitro findings were in keeping with the in vivo results.
$H D L-E C$ turnover. The decay curves of HDL-EC specific activity are depicted in Fig. 3, and the kinetic parameters are given in the Appendix. They were obtained after subtraction of the in vivo esterification of free cholesterol. The correction for continuing esterification of cholesterol increased with time: it represented $6.4 \%$ of the HDL-EC radioactivity in the controls and $11 \%$ in the alcohol-fed animals during the first 2 h. Only during the last $5 \mathrm{~h}$ did it increase up to $35 \%$ and $59 \%$, respectively. This correction factor did not affect significantly the differences in the kinetics of the rapidly exchangeable pool between alcohol-fed and control animals.

The specific activity of HDL-EC decayed biexponentially in both groups of animals. After $10 \mathrm{~min} 102.2 \pm 6.2 \%$ of the esterified cholesterol injected was recovered in plasma lipoproteins, indicating the absence of rapid sequestration. Also, $<1 \%$ of the tritiated cholesterol injected was found in the liver of seven baboons in which needle biopsy tissue specimens were obtained 5-8 h after injection of labeled HDL. The biexponential clearance without evidence of rapid sequestration and minimal hepatic uptake of HDL-EC suggest that the preparation behaved as native HDL (41).

In ethanol-fed baboons, the slope of the rapid exponential tended to decrease, whereas that of the slow phase increased. The kinetics of the rapidly exchangeable pool of HDL-EC most likely reflects transfer of esterified cholesterol from plasma HDL to lipoproteins in the VLDL-LDL pathway. The size of this pool was equivalent to $67.5 \%$ and $56 \%$ of the plasma HDL-EC in alcohol-fed and control animals, respectively. The rate constant of removal of HDL-EC decreased in ethanol-fed baboons to approximately half of that of controls. However,

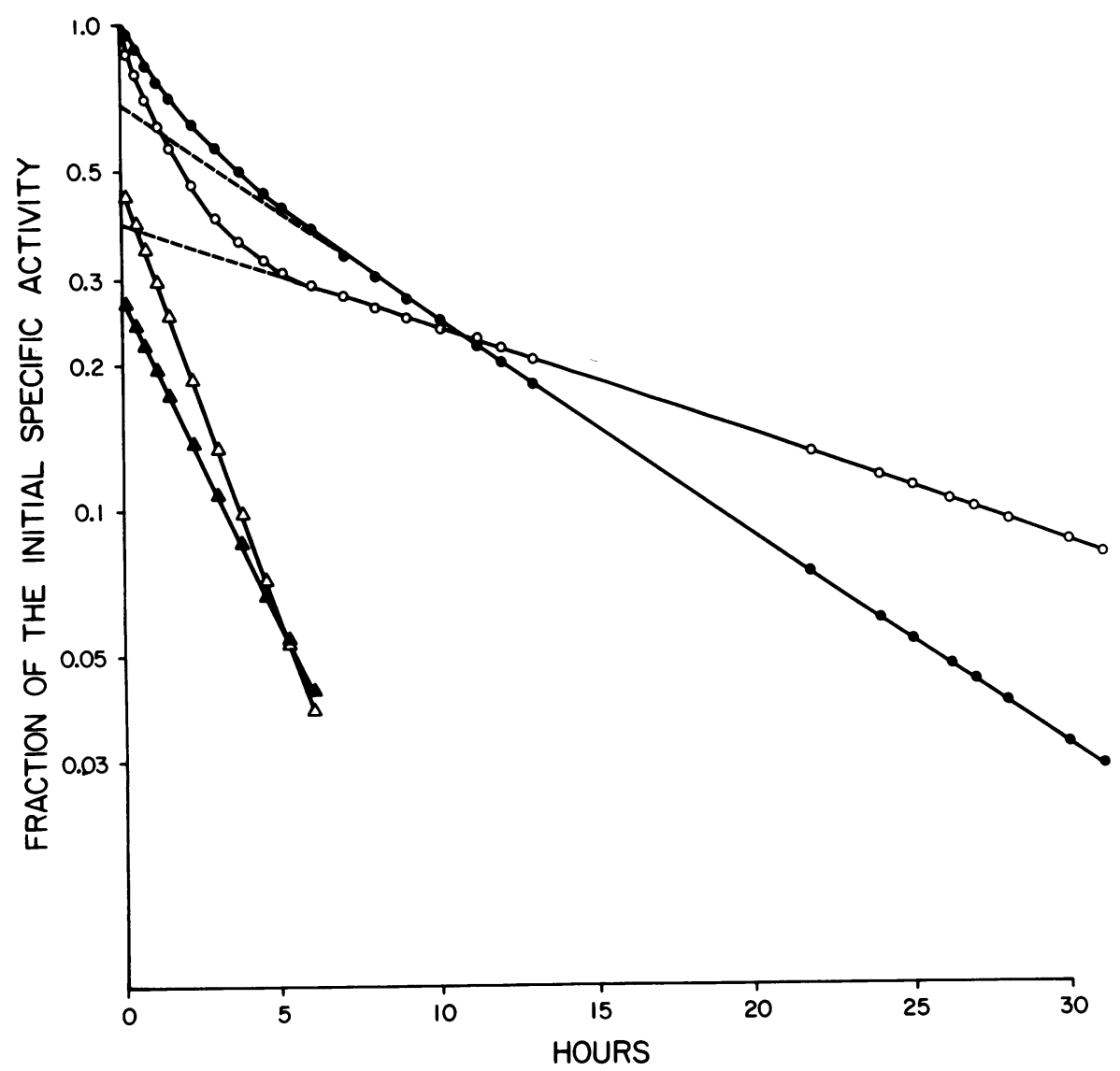

Figure 3. Average decay curve of esterified cholesterol specific activity in plasma HDL as a fraction of the 2-min values in five pairs of baboons fed either ethanolcontaining or control diets. Baboons were injected with autologous HDL doubly labeled in vitro with ${ }^{14} \mathrm{C}$ in free cholesterol and ${ }^{3} \mathrm{H}$ in both free and esterified cholesterol. The specific activity of HDL-EC (circles) was obtained after subtraction of the in vivo esterification of $\left[{ }^{3} \mathrm{H}\right]$ free cholesterol (assessed by the appearance of $\left[{ }^{14} \mathrm{C}\right]$ free cholesterol in the esterified fraction). The rapid exponential (triangles) was calculated by subtraction of the second exponential from the experimental values. There was a decreased slope of the first exponential and an increase slope of the second exponential in the alcohol-fed animals. This was reflected in a significant decrease in the rate constant of removal of HDL-esterified cholesterol (see Appendix). Ethanol-fed baboons: •, experimental values; $\Delta$, calculated values. Pair-fed controls: $O$, experimental values; $\Delta$, calculated values. 
the total catabolic rate was not significantly affected, because of the larger pool of HDL-EC in alcohol-fed animals. Also, there was no significant change in the production rate of HDLEC. The decreased rate constant of removal of HDL-EC was associated with differences in the appearance of the radioactive EC into LDL (Fig. 4): the maximal specific activity of LDLEC in the controls was reached in 3 hours and, after this time, the values were similar to those in HDL. By contrast, in the alcohol-fed animals, the maximal radioactivity of LDL-EC was delayed ( $5 \mathrm{~h}$ vs. $3 \mathrm{~h}$ ); it was also significantly lower than in controls $(27 \pm 1 \%$ of the initial HDL-EC specific activity vs. $44 \pm 8 \%$ in the controls; $P<0.05$ ) and after reaching maximal levels, it took $>10 \mathrm{~h}$ for the LDL-EC values to become similar to those in HDL.

Splanchnic removal or production of serum cholesterol. Whereas no net extraction or release of cholesterol was detected in control baboons during a single passage through the splanchnic vascular bed, significantly more extraction than release of cholesterol was found in the alcohol-fed animals (Table II). There were no differences in plasma volume, hepatic blood flow, or hematocrit values between these two types of animals (Table III). Significant extraction of esterified cholesterol was found in IDL and LDL and of free cholesterol in HDL of the alcohol-fed animals. There were no detectable changes in cholesterol concentration between arterial and portal blood during laparotomy in the three pairs of baboons in which this was measured.

Fecal excretion of endogenous steroids. The fecal excretion of endogenous neutral steroids doubled in the ethanol-fed baboons (Table IV). The recovery of the inert fecal marker $\left(\mathrm{Cr}_{2} \mathrm{O}_{3}\right)$ was similar in alcohol-fed baboons $(54 \pm 9 \%)$ and in pair-fed controls $(57 \pm 5 \%)$. The output of acidic steroids was

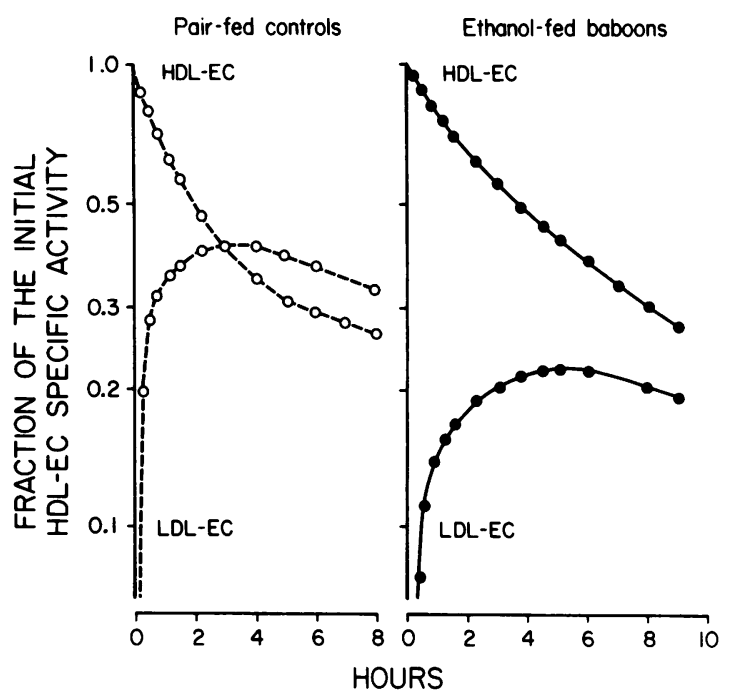

Figure 4. Relationships between the decay in HDL-EC and the increase in LDL-EC specific activities. HDL-EC (labeled in vitro) was injected in baboons fed either ethanol-containing or control diet. Specific activities are expressed as a fraction of the 2-min value in HDL-EC. In controls, the increasing LDL-EC specific activities crossed over the decreasing HDL-EC specific activities, suggesting a precursor-product relationship. In alcohol-fed animals, the increase in LDL-EC specific activities was smaller and did not cross over HDLEC specific activities, indicating that a significant amount of unlabeled esterified cholesterol enters LDL from a source other than HDL. increased by alcohol feeding in only four of the five pairs, and the difference was not statistically significant. Thus, the alcoholfed animals excreted in the feces an excess of neutral and acidic steroids equivalent to $1.7 \pm 0.4 \mu \mathrm{mol}$ of cholesterol per $\mathrm{kg}$ body weight per hour, a value that was comparable with the increase in the production rate of serum free cholesterol.

\section{Discussion}

The present study revealed that the elevations of the free and esterified fractions of HDL-cholesterol after chronic alcohol consumption result from two independent mechanisms with opposite significance with regard to tissue cholesterol mobilization. The major increase of HDL-cholesterol was found in the esterified fraction. This was associated with decreased turnover. By contrast, free cholesterol mobilization from peripheral tissues and its biliary excretion appeared to be enhanced.

Chronic administration of diets containing 50\% of energy as ethanol to baboons produces sustained hyperlipemia (42). A similar hyperlipemia (although of a lesser magnitude) was produced in human volunteers given only $20-30 \%$ of energy as alcohol (43). Under these baboons' conditions, cholesterol increases in several lipoprotein fractions. Previous studies in rats $(8,44)$ and baboons $(42)$ indicate that ethanol feeding increases hepatic release in VLDL. These effects are associated with elevation of free cholesterol in VLDL, IDL, and LDL and esterified cholesterol in VLDL and IDL (Table I). The major increase (in absolute terms) occurred in HDL and included both free and esterified cholesterol. This alteration was particularly prominent at early stages of alcoholic liver injury (fatty liver with or without perivenular fibrosis), whereas the hypercholesterolemia decreased or disappeared in ethanolfed baboons with septal fibrosis or cirrhosis. Therefore, the present study on the mechanisms of the hypercholesterolemia was limited to animals with early stages of alcoholic liver injury.

Regarding cholesterol esters, its removal rate from HDL was significantly decreased, after chronic alcohol feeding. This was associated with a slower and smaller increment of the cholesterol ester specific activity in LDL (Fig. 4). Moreover, whereas in control baboons the latter crossed over the decreasing specific activity of the esters in HDL (suggesting a precursorproduct relationship), in the alcohol-fed baboons, no such crossover occurred, indicating that a significant fraction of unlabeled esters had entered LDL from a source other than direct transfer from HDL. This may reflect either a decreased transfer of esterified cholesterol from HDL to VLDL and IDL or an increase release of esterified cholesterol in nascent VLDL, either of intestinal or hepatic origin. We did not find net output of VLDL-EC in the hepatic venous blood (Table II), but an intestinal source cannot be excluded without lymph measurements. In fact, an increased intestinal or hepatic release of esterified cholesterol in the VLDL-LDL pathway may slow down esterified cholesterol transfer from HDL. Whether ethanol consumption affects the esterified cholesterol transfer/exchange protein or alters the properties of the donor or the recipient lipoproteins in this transfer process remains to be determined. In spite of the slower rate of transfer, a normal flux of cholesterol esters through this pathway was maintained because of the enlargement of the plasma HDLEC pool. 


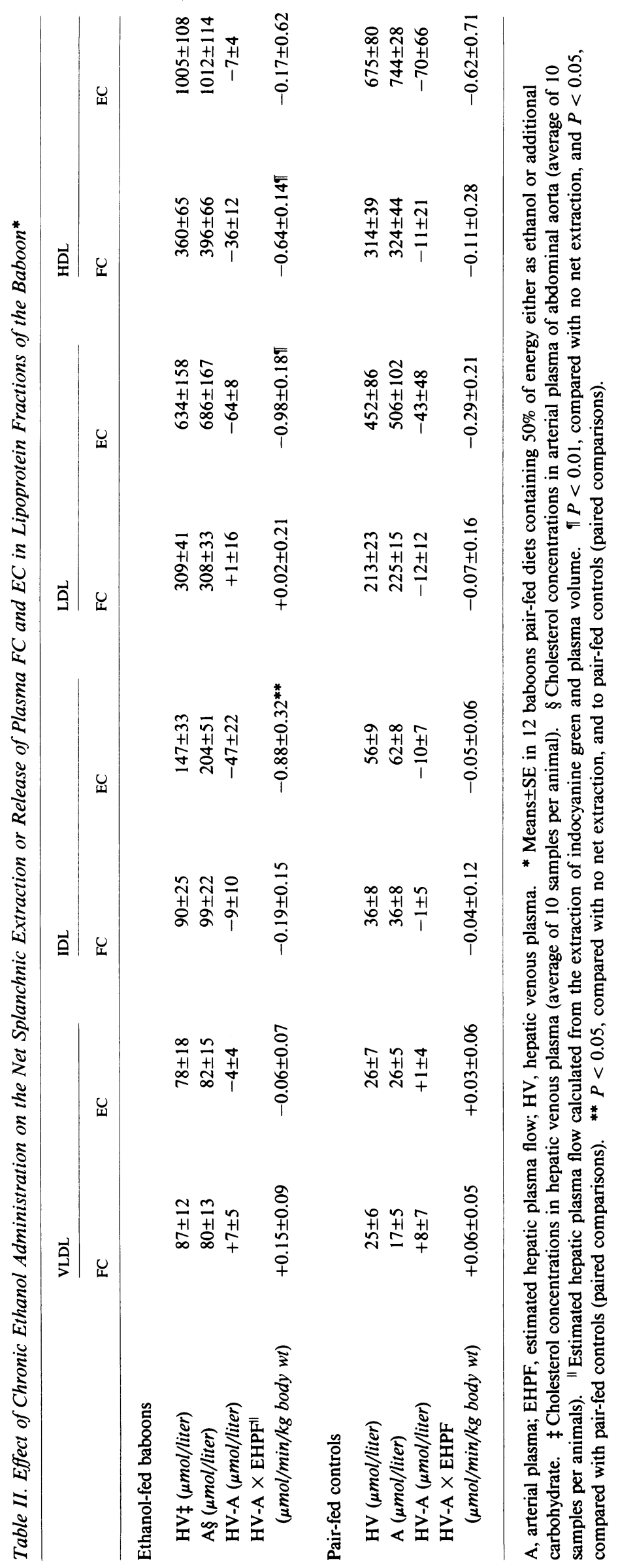


Table III. Effects of Chronic Ethanol Feeding on Hemodynamic Parameters*

\begin{tabular}{llll}
\hline & $\begin{array}{l}\text { Ethanol-fed } \\
\text { baboons }\end{array}$ & $\begin{array}{l}\text { Pair-fed } \\
\text { controls }\end{array}$ & $P \ddagger$ \\
\hline $\begin{array}{l}\text { Body weight }(\mathrm{kg}) \\
\begin{array}{c}\text { Plasma volume } \\
(\mathrm{ml} / \mathrm{kg})\end{array}\end{array}$ & $10.9 \pm 0.5$ & $12.3 \pm 2.0$ & $<0.05$ \\
$\begin{array}{c}\text { Arterial hematocrit } \\
(\%)\end{array}$ & $47.4 \pm 7.3$ & $45.2 \pm 5.2$ & $\mathrm{NS}$ \\
$\begin{array}{c}\text { Hepatic venous } \\
\text { hematocrit (\%) }\end{array}$ & $39.5 \pm 1.2$ & $42.1 \pm 0.7$ & $\mathrm{NS}$ \\
$\begin{array}{c}\text { Hepatic blood flow } \\
(\mathrm{ml} / \mathrm{kg} / \mathrm{min})\end{array}$ & $37.9 \pm 1.9$ & $41.1 \pm 0.6$ & $\mathrm{NS}$ \\
\hline
\end{tabular}

* Mean \pm SE of values in 10 baboons pair-fed $50 \%$ of energy either as ethanol or as additional carbohydrate.

$\ddagger$ Paired comparisons.

At these early stages of alcoholic liver damage, alcohol feeding was associated with an apparent increase in L-CAT activity both in vitro and in vivo. In vitro, this increase was accounted for by the increased pool of plasma HDL-FC in alcohol-fed baboons; as a consequence, the fractional conversion rate was similar to that of controls. In vivo, the increased rate of esterification was also proportional to the HDL-FC production rate, namely to the input of free cholesterol becoming available for esterification, suggesting that the transferase activity per se is not affected. It has been hypothesized that the transfer of esterified cholesterol is structurally and functionally coupled with L-CAT activity in a common lipoprotein complex (45). Therefore, one would expect the decreased transfer to be associated with decreased esterification, but this was not the case. In fact, the maintenance of a high esterification rate in the face of a decreased transfer rate appears to be one of the major reasons for the increased HDL-esterified cholesterol levels in the alcohol-fed animals.

Contrasting with its effect on esterified cholesterol, alcohol consumption increased the total amount of cholesterol being turned over as HDL-FC. Part of this increase can be accounted for by increased esterification. But HDL-FC exchange/transfer might also provide a pathway for increased mobilization of tissue cholesterol for excretion into the bile.

HDL-cholesterol could derive from tissue cholesterol (36) or from cholesterol of other lipoproteins originating in the liver or the intestine. Since the splanchnic territory both

Table IV. Effects of Chronic Ethanol Administration on Fecal Excretion of Endogenous Steroids*

\begin{tabular}{|c|c|c|c|}
\hline & Neutral steroids & Acidic steroids & Total steroids \\
\hline & $\underset{w t / d}{\mu m o l / k g ~ b o d y}$ & $\underset{w t / d}{\mu m o l / k g}$ body & $\underset{w t / d}{\mu m o l / k g ~ b o d y}$ \\
\hline $\begin{array}{c}\text { Ethanol-fed } \\
\text { baboons }\end{array}$ & $46.0 \pm 10.7$ & $61.8 \pm 12.7$ & $107.7 \pm 13.5$ \\
\hline $\begin{array}{l}\text { Pair-fed } \\
\text { controls }\end{array}$ & $19.7 \pm 5.7$ & $46.1 \pm 4.5$ & $65.9 \pm 7.3$ \\
\hline$P \ddagger$ & $<0.05$ & NS & $<0.01$ \\
\hline
\end{tabular}

* Mean \pm SEM of 10 baboons pair-fed cholesterol-free diets containing $50 \%$ of energy either as ethanol or addition carbohydrate for 2 yr. $\ddagger$ Paired comparisons. releases and removes plasma cholesterol, we determined the net balance between these two processes by measuring hepatic plasma flow and the cholesterol concentrations in arterial and hepatic venous plasma. Whereas no net changes were found in controls, in the alcohol-fed animals, there was net splanchnic extraction of cholesterol. This was mainly due to rapid extraction of cholesterol by the liver since there were no arterioportal venous differences in cholesterol concentrations. Thus, more cholesterol was available for excretion in the bile. The source of cholesterol extracted by the liver cannot be established with certainty. The predominance of hepatic extraction over release suggests that the hypercholesterolemia must originate extrahepatically. A likely source is mobilization of tissue cholesterol. An alternate source could be cholesterol entering the circulation through the thoracic duct.

The plasma cholesterol removed by the splanchnic territory of the alcohol-fed animals could be excreted in the bile and lost in the feces, or it could recirculate in other lipoproteins or could accumulate in the liver. Our results show a significant increase in the fecal excretion of steroids of endogenous (rather than dietary) origin. Moreover, the isotopic balance method used in this study does not include the cholesterol newly synthesized in the liver that is excreted in the bile without previous circulation in plasma lipoproteins. Under our conditions, the greatest increase in the feces occurred in the form of neutral steroids. Acidic steroids also showed a trend toward an increase of a lesser magnitude. These findings are in keeping with studies in other species. Nestel et al. (46) found that ethanol intake increased fecal excretion of acidic steroids in three hyperlipemic alcoholics and in some normolipemic subjects given a small alcohol dose for $8 \mathrm{~d}$. Chronic administration of ethanol (20-30\% of calories) produced no significant changes in fecal steroid excretion (43). Two recent reports, one in rats given $36 \%$ of energy as ethanol in cholesterol-free diet (47) and the other in pigs fed diets containing both cholesterol and ethanol ( $25 \%$ of energy) (48) also showed increased fecal excretion of steroids. Whereas in rats the major excretion was as neutral steroids, in pigs, the increase was as acidic steroids. Thus, ethanol consumption promotes loss of cholesterol in the feces; differences in species or in diet may account for the type of steroid that predominates.

Kinetic studies using cholesterol-labeled lipoproteins (25) indicate that the major precursor of biliary cholesterol and bile acids is HDL-FC. In the alcohol-fed baboons, there was net splanchnic extraction of free cholesterol from HDL. The increased fecal excretion after alcohol can be accounted for by the difference in the production rate of new (nonrecycled) HDL-FC between alcohol-fed and control baboons. Most of the remaining cholesterol removed in the splanchnic territory must recirculate through the blood or the lymphatic pathway, since we found only minimal cholesterol accumulation in the liver, contrary to our previous observations in the rat (49). Preliminary results in volunteers given alcohol are consistent with the observations in the baboon rather than in the rat (unpublished observation). In addition to the extraction of HDL-FC, there was also net splanchnic extraction of esterified cholesterol from IDL and LDL in the alcohol-fed animals. The source of this cholesterol is unknown. Net splanchnic extraction of LDL-EC has also been found in patients undergoing diagnostic cardiac catheterization for valvular or ischemic heart disease (50). The splanchnic extraction of LDL-EC, found in human patients (50), was also seen in the alcohol- 
fed animals, but it was not apparent in our control baboons. In the alcohol-fed baboons the splanchnic extraction is considerably greater than the fecal excretion of steroids, suggesting a large recirculation. Our findings indicate that the transfer of esterified cholesterol from HDL to the VLDL-LDL pathway is not increased. The increased output of VLDL-cholesterol into the hepatic vein is insufficient to account for the increased cholesterol extraction from catabolic products of VLDL, but the lymphatic contribution to blood cholesterol was not measured. The thoracic lymph output of endogenous cholesterol in primates (51) is four- to fivefold greater than the fecal excretion of steroids (11); an increase in the lymphatic circulation by alcohol could account for the dissociation between splanchnic extraction and fecal excretion of steroids. The lymph includes cholesterol of intestinal origin. It has also been postulated that because of their large size, the VLDL particles produced by the alcohol-fed baboons (40) may be impeded in their passage into the sinusoids through the fenestrations of the hepatic endothelial cells, which are decreased in number (52). As a consequence, the VLDL particles may be transported from the Disse's space into the hepatic lymphatics rather than into the hepatic vein.

The decreased transfer of esterified cholesterol from HDL to LDL and the enhanced hepatic uptake of esterified cholesterol from IDL and LDL tend to deplete LDL-EC. LDL is one of the main sources of tissue cholesterol and the factor most incriminated in the genesis of atherosclerosis. However, the total amount of LDL-EC was not decreased. Thus, the availability of plasma LDL-EC for the cell probably remained unaffected. By contrast, the ethanol-induced alterations of free cholesterol metabolism could facilitate cholesterol efflux from cells to plasma HDL, followed by excretion in the bile. The increase in daily excretion of cholesterol $(\sim 50 \mu \mathrm{mol} / \mathrm{kg}$ of body weight), over prolonged periods of time, could counteract cholesterol accumulation in tissues, to the extent that it is not compensated by a concomitant increase in synthesis. Thus, the effects of ethanol on free cholesterol may contribute to the reported prevention of atherosclerosis after alcohol consumption.

\section{Appendix}

Kinetics of HDL-cholesterol turnover. Kinetic parameters were calculated from semilogarithmic plots of the specific activities of $\mathrm{HDL}-\left[{ }^{14} \mathrm{C}\right]$ free cholesterol and $-\left[{ }^{3} \mathrm{H}\right]$ esterified cholesterol versus time. Since each curve could be resolved into two exponential functions, the kinetics were analyzed as a two-pool model (30). This model postulates two pools (A and B) exchanging freely, with input occurring predominantly into pool A. The analysis provides the half lives $\left(t_{1 / 2}\right)$ and the slopes $(\alpha$ and $\beta$ ) of the two exponentials and permits estimation of the size of pool $A$ and the rates of production, transfer, and removal of cholesterol. The size of the pool $\mathrm{A}\left(M_{\mathrm{A}}\right)$ was calculated by dividing the radioactivity injected $\left(R_{\mathrm{A}}\right)$ by the sum of the intercepts of the rapid $\left(C_{\mathrm{A}}\right)$ and slow $\left(C_{\mathrm{B}}\right)$ exponentials of the specific activity decay curves with the ordinate. The size of pool B cannot be calculated. The removal rates constants $\left(-k_{\mathrm{AA}}\right.$ and $\left.-k_{\mathrm{BB}}\right)$ reflect irreversible losses as well as transfer between pools A and B. The total amount of plasma cholesterol being turned over, namely the total catabolic rate (TCR), was considered to be the product of $-k_{\mathrm{AA}}$ by the size of pool $\mathrm{A}$. The rate constants $k_{\mathrm{BA}}$ (transfer rate from pool $\mathrm{B}$ to pool $\mathrm{A}$ ), $k_{\mathrm{A}}$ (irreversible removal rate from pool $\mathrm{A})$ and $k_{\mathrm{AB}}$ (transfer rate from pool $\mathrm{A}$ to pool $\mathrm{B}$ ) can be calculated if the irreversible removal rate from pool $\mathrm{B}\left(k_{\mathrm{B}}\right)$ is assumed to be equal to zero during the time of the study. The production rate $\left(P R_{\mathrm{A}}\right)$,
Table V. Kinetic Parameters* of HDL-Cholesterol Turnover

\begin{tabular}{|c|c|c|c|}
\hline & $\begin{array}{l}\text { Ethanol-fed } \\
\text { baboons }\end{array}$ & $\begin{array}{l}\text { Pair-fed } \\
\text { controls }\end{array}$ & $P \S$ \\
\hline \multicolumn{4}{|l|}{$\begin{array}{l}\text { HDL-free } \\
\text { cholesterol }\end{array}$} \\
\hline \multicolumn{4}{|l|}{$R_{\mathrm{A}}\left(d p m \times 10^{-3} /\right.$} \\
\hline$C_{\mathrm{A}}(d p m / \mu m o l)$ & $2886 \pm 1105$ & $\begin{array}{l}19.4 \pm 0.9 \\
2190 \pm 726\end{array}$ & NS \\
\hline$C_{\mathrm{B}}(\mathrm{dpm} / \mu \mathrm{mol})$ & $524 \pm 219$ & $479 \pm 243$ & NS \\
\hline \multicolumn{4}{|l|}{$M_{\mathrm{A}}(\mu \mathrm{mol} / \mathrm{kg}$} \\
\hline body wt) & $16.6 \pm 4.3$ & $9.6 \pm 4.0$ & NS \\
\hline$t_{\mathrm{A} / 2}(h)$ & $0.32 \pm 0.05$ & $0.41 \pm 0.08$ & NS \\
\hline$t_{\mathrm{B} / 2}(h)$ & $24.2 \pm 5.3$ & $21.9 \pm 3.2$ & NS \\
\hline$\alpha\left(h^{-I}\right)$ & $2.4 \pm 0.5$ & $2.0 \pm 0.4$ & NS \\
\hline$\beta\left(h^{-1}\right)$ & $0.034 \pm 0.007$ & $0.036 \pm 0.008$ & NS \\
\hline$k_{A A}\left(h^{-1}\right)$ & $2.0 \pm 0.3$ & $1.7 \pm 0.4$ & NS \\
\hline TCR $(\mu \mathrm{mol} / \mathrm{kg} / \mathrm{h})$ & $34.77 \pm 11.72$ & $13.82 \pm 4.13$ & $<0.05$ \\
\hline$P R_{\mathrm{A}}(\mu \mathrm{mol} / \mathrm{kg} / \mathrm{h})$ & $3.0 \pm 1.0$ & $1.4 \pm 0.8$ & $<0.05$ \\
\hline$k_{\mathrm{BB}}\left(h^{-1}\right)$ & $0.45 \pm 0.11$ & $0.33 \pm 0.06$ & NS \\
\hline$k_{\mathrm{A}}\left(h^{-1}\right)$ & $0.19 \pm 0.03$ & $0.20 \pm 0.03$ & NS \\
\hline$k_{\mathrm{AB}}\left(h^{-1}\right)$ & $1.82 \pm 0.33$ & $1.49 \pm 0.38$ & NS \\
\hline \multicolumn{4}{|l|}{$\begin{array}{l}\text { HDL-esterified } \\
\text { cholesterol }\end{array}$} \\
\hline \multicolumn{4}{|l|}{$R_{\mathrm{A}}\left(d p m \times 10^{-3}\right)$} \\
\hline$k g$ body $w t)$ & $783 \pm 149$ & $487 \pm 100$ & NS \\
\hline$C_{\mathrm{A}}(d p m / \mu m o l)$ & $6031 \pm 2223$ & $8116 \pm 2016$ & NS \\
\hline$C_{\mathbf{B}}(d p m / \mu m o l)$ & $15526 \pm 1928$ & $12525 \pm 4218$ & NS \\
\hline \multicolumn{4}{|l|}{$M_{\wedge}(\mu \mathrm{mol} / \mathrm{kg}$} \\
\hline body wt) & $32.6 \pm 10.3$ & $20.4 \pm 6.5$ & NS \\
\hline$t_{A y_{2}}(h)$ & $1.56 \pm 0.45$ & $0.90 \pm 0.16$ & NS \\
\hline$t_{\mathrm{B} / 2}(h)$ & $6.90 \pm 1.2$ & $13.60 \pm 1.60$ & 0.01 \\
\hline$\alpha\left(h^{-1}\right)$ & $0.54 \pm 0.08$ & $0.85 \pm 0.12$ & NS \\
\hline$\beta\left(h^{-1}\right)$ & $0.10 \pm 0.01$ & $0.05 \pm 0.01$ & $<0.02$ \\
\hline$k_{\mathrm{AA}}\left(h^{-1}\right)$ & $0.22 \pm 0.04$ & $0.40 \pm 0.06$ & $<0.05$ \\
\hline TCR $(\mu \mathrm{mol} / \mathrm{kg} / \mathrm{h})$ & $6.9 \pm 1.4$ & $6.9 \pm 1.9$ & NS \\
\hline$P R_{\mathrm{A}}(\mu \mathrm{mol} / \mathrm{kg} / \mathrm{h})$ & $3.9 \pm 1.4$ & $3.5 \pm 1.2$ & NS \\
\hline$k_{\mathrm{BB}}\left(h^{-1}\right)$ & $0.42 \pm 0.07$ & $0.53 \pm 0.13$ & NS \\
\hline
\end{tabular}

TCR, total catabolic rate.

* Defined in Appendix.

¥ Mean \pm SE of values in 10 baboons pair-fed $50 \%$ of energy either as ethanol or as additional carbohydrate.

§ Paired comparisons.

indicating the entry of new cholesterol into the pool A (excluding reentry from other pools), as well as the other rates, were calculated according to Gurpide et al. (30).

The kinetic parameters derived from the two-pool analysis of the individual curves are given in Table $\mathrm{V}$. The dose injected $\left(R_{\mathrm{A}}\right)$ to the alcohol-fed baboons tended to be higher than that of controls, because of the increase in serum-free cholesterol (available for in vitro exchange) and the enhanced rate of esterification.

The kinetics is consistent with a model in which HDL-free cholesterol enters a more rapidly exchangeable pool $A$ in equilibrium with a slower turning over pool B. By contrast, HDL-EC appears to enter a large and slowly turning over pool $A$ in equilibrium with a probably smaller and more rapidly turning over pool $B$. The transfer rates of HDL-EC between pools $A$ and $B$ were not calculated because the assumption that there is no irreversible loss from pool B is not tenable. As in the case of other kinetic studies, the identity of the processes contributing to each pool remains speculative.

\section{Acknowledgments}

The expert technical assistance of Mr. Lawrence Tannenbaum, Ms. Frances Finkelman, and Mr. Bruce Seabrook is gratefully acknowledged.

This study was supported, in part, by grant AA-03508 from the U. S. Public Health Service and by the Veterans Administration. 


\section{References}

1. Yano, K., G. G. Rhoads, and A. Kagan. 1977. Coffee, alcohol and risk of coronary heart disease among Japanese men living in Hawaii. N. Engl. J. Med. 297:405-409.

2. Rudel, L. L., C. W. Leathers, M. G. Bond, and B. C. Bullock. 1981. Dietary ethanol induced modifications in hyperlipoproteinemia and atherosclerosis in nonhuman primates (Macaca nemestrina). $\mathrm{Ar}$ teriosclerosis. 1:144-155.

3. Miller, N. E., P. J. Nestel, and P. Clifton-Bligh. 1976. Relationship between plasma lipoprotein cholesterol concentrations and the pool size and metabolism of cholesterol in man. Atherosclerosis. 23:535547.

4. Nestel, P. J., and N. E. Miller. 1980. Cholesterol kinetics and fecal steroid excretion in subjects with primary hyperalphalipoproteinemia. Atherosclerosis. 36:127-134.

5. Stein, O., J. Vanderhoek, and Y. Stein. 1976. Cholesterol content and sterol synthesis in human skin fibroblasts and rat aortic smooth muscle cells exposed to lipoprotein-depleted serum and high density apoprotein/phospholipid mixtures. Biochim. Biophys. Acta. 431:347358.

6. Rothblat, G. H., and M. C. Phillips. 1982. Mechanism of cholesterol efflux from cells. Effects of acceptor structure and concentration. J. Biol. Chem. 257:4775-4782.

7. Johansson, B. G., and C. B. Laurell. 1969. Disorders of serum $\alpha$-lipoproteins after alcoholic intoxication. Scand. J. Clin. Lab. Invest. 23:231-233.

8. Baraona, E., and C. S. Lieber. 1970. Effects of chronic ethanol feeding on serum lipoprotein metabolism in the rat. J. Clin. Invest. 49:769-778.

9. Castelli, W. P., J. T. Doyle, T. Gordon, C. G. Hames, M. C. Hjortland, S. B. Hulley, A. Kagan, and W. J. Zukel. 1977. Alcohol and blood lipids. The Cooperative Lipoprotein Phenotyping Study. Lancet. 2:153-155.

10. Chapman, M. J. 1980. Animal lipoproteins: chemistry, structure and comparative aspects. J. Lipid Res. 21:789-853.

11. Eggen, D. A. 1974. Cholesterol metabolism in rhesus monkey, squirrel monkey and baboon. J. Lipid Res. 15:139-145.

12. Strong, J. P., D. A. Eggen, W. P. Newman, and R. D. Martinez. 1968. Naturally occurring and experimental atherosclerosis in primates. Ann. N.Y. Acad. Sci. 149:882-894.

13. Lieber, C. S., and L. M. DeCarli. 1976. Animal models of ethanol dependence and liver injury in rats and baboons. Fed. Proc. 35:1232-1236.

14. Duritz, G., and E. B. Truitt. 1964. A rapid method for the simultaneous determination of acetaldehyde and ethanol in blood using gas chromatography. Q. J. Stud. Alcohol. 25:498-510.

15. Walk, L. 1961. Roentgenologic determination of the liver volume. Acta Radiol. 55:49-56.

16. Folch, J., M. Lees, and G. H. Sloane-Stanley. 1957. A simple method for the isolation and purification of total lipids from animal tissues. J. Biol. Chem. 226:497-509.

17. Amenta, J. S. 1964. A rapid chemical method for quantification of lipids separated by thin-layer chromatography. J. Lipid Res. 5:270272.

18. Snyder, F., and N. Stephens. 1959. A simplified spectrophotometric determination of ester groups in lipids. Biochem. Biophys. Acta. 34:244-245.

19. Searcy, R. L., and L. M. Bergquist. 1960. A new color reaction for the quantitation of serum cholesterol. Clin. Chim. Acta. 5:192199.

20. Zak, B., and E. Epstein. 1961. New cholesterol reagent. Clin. Chem. 7:268-270.

21. Allain, C. C., L. S. Poon, C. S. G. Chan, W. Richmond, and P. C. Fu. 1974. Enzymatic determination of total serum cholesterol. Clin. Chem. 20:470-475.
22. Rudel, L. L., and M. D. Morris. 1973. Determination of cholesterol using o-phthalaldehyde. J. Lipid Res. 14:364-366.

23. Wahlefeld, A. W., 1974. Triglyceride determination after enzymatic hydrolysis. In Methods of Enzymatic Analysis. Vol. 4. H. U. Bergmeyer, editor. Verlag Chemie GmbH, Weinheim, Germany. 18811835.

24. Stokke, K. T., and K. R. Norum. 1971. Determination of lecithin: cholesterol acyltransferase in human blood plasma. Scand. $J$. Clin. Lab. Invest. 27:21-27.

25. Schwartz, C. C., Z. R. Vlahcevic, L. G. Halloran, and L. Swell. 1981. An in vivo evaluation in man of the transfer of esterified cholesterol between lipoproteins and into the liver and bile. Biochim. Biophys. Acta. 663:143-162.

26. Chung, B. H., T. Wilkinson, J. C. Geer, and J. P. Segrest. 1980. Preparative and quantitative isolation of plasma lipoproteins: rapid, discontinuous density gradient ultracentrifugation in a vertical rotor. J. Lipid Res. 21:284-291.

27. Bronzert, T. J., and H. B. Brewer. 1977. New micromethod for measuring cholesterol in plasma lipoprotein fractions. Clin. Chem. 23:2089-2098.

28. Noble, R. P. 1968. Electrophoretic separation of plasma lipoproteins in agarose gel. J. Lipid Res. 9:693-700.

29. Fainaru, M., R. J. Havel, and K. Imaizumi. 1977. Apoprotein content of plasma lipoproteins of the rat separated by gel chromatography or ultracentrifugation. Biochem. Med. 17:347-355.

30. Gurpide, E., J. Mann, and E. Sandberg. 1964. Determination of kinetic parameters in a two-pool system by administration of one or more tracers. Biochemistry. 3:1250-1255.

31. Goodman, D. S., and R. P. Noble. 1968. Turnover of plasma cholesterol in man. J. Clin. Invest. 47:231-241.

32. Wilson, J. D. 1970. The measurement of the exchangeable pools of cholesterol in the baboon. J. Clin. Invest. 49:655-665.

33. Schwartz, C. C., Z. R. Vlahcevic, M. Berman, J. G. Meadows, R. M. Nisman, and L. Swell. 1982. Central role of high density lipoprotein in plasma free cholesterol metabolism. J. Clin. Invest. 70: 105-116.

34. Gregersen, M. I. 1944. A practical method for the determination of blood volume with the dye T-1824. J. Lab. Clin. Med. 29:12661286.

35. Caesar, J., S. Shaldon, L. Chiandussi, L. Guevara, and S. Sherlock. 1961. The use of indocyanine green in the measurement of hepatic blood flow and as a test of hepatic function. Clin. Sci. 21:4357.

36. Grundy, S. M., and E. H. Ahrens. 1966. An evaluation of the relative merits of two methods for measuring the balance of sterols in man: isotopic balance versus chromatographic analysis. $J$. Clin. Invest. 45:1503-1515.

37. Davignon, J., W. J. Simmonds, and E. H. Ahrens. 1968. Usefulness of chromic oxide as an internal standard for balance studies in formula-fed patients and for assessment of colonic function. J. Clin. Invest. 47:127-138.

38. Miettinen, T. A., E. H. Ahrens, and S. M. Grundy. 1965. Quantitative isolation and gas-liquid chromatographic analysis of total dietary and fecal neutral steroids. J. Lipid Res. 6:411-424.

39. Grundy, S. M., E. H. Ahrens, and T. A. Miettinen. 1965. Quantitative isolation and gas-liquid chromatographic analysis of total fecal bile acids. J. Lipid Res. 6:397-410.

40. Snedecor, G. W., and W. G. Cochran. 1967. Statistical Methods. 6th edition. The Iowa State University Press. Ames, Iowa.

41. Van't Hoof, F., and R. J. Havel. 1981. Metabolism of chromatographically separated rat serum lipoproteins specifically labeled with ${ }^{125}$ I-apoprotein E. J. Biol. Chem. 256:3963-3968.

42. Baraona, E., M. Savolainen, C. Karsenty, and C. S. Lieber. 1984. Pathogenesis of alcoholic hypertriglyceridemia and hypercholesterolemia. Trans. Assoc. Am. Phys. 35:285-299.

43. Crouse, J. R., and S. M. Grundy. 1984. Effects of alcohol on 
plasma lipoproteins and cholesterol and triglyceride metabolism in man. J. Lipid Res. 25:486-496.

44. Baraona, E., R. C. Pirola, and C. S. Lieber. 1973. Pathogenesis of postprandial hyperlipemia in rats fed ethanol-containing diets. $J$. Clin. Invest. 52:296-303.

45. Fielding, P. E., and C. J. Fielding. 1980. A cholesteryl ester transfer complex in human plasma. Proc. Natl. Acad. Sci. USA. 77: 3327-3330.

46. Nestel, P. J., L. A. Simons, and Y. Homma. 1976. Effects of ethanol on bile acid and cholesterol metabolism. Am. J. Clin. Nutr. 29:1007-1015.

47. Cohen, B. I., and R. F. Raicht. 1981. Sterol Metabolism in the rat: effects of alcohol on sterol metabolism in two strains of rats. Alcohol. Clin. Exp. Res. 5:225-229.

48. Topping, D. L., R. A. Weller, C. J. Nader, J. D. Calvert, and
R. J. Illman. 1982. Adaptive effects of dietary ethanol in the pig: changes in plasma high-density lipoproteins and fecal steroid excretion and mutagenicity. Am. J. Clin. Nutr. 36:245-250.

49. Lefevre, A. F., L. M. DeCarli, and C. S. Lieber. 1972. Effect of ethanol on cholesterol and bile acid metabolism. J. Lipid Res. 13: 48-55.

50. Sniderman, A., D. Thomas, D. Marpole, and B. Teng. 1978. Low density lipoprotein. A metabolic pathway for return of cholesterol to the splanchnic bed. J. Clin. Invest. 61:867-873.

51. Klein, R. L., and L. L. Rudel. 1983. Cholesterol absorption and transport in thoracic duct lymph lipoproteins of nonhuman primates. Effect of dietary cholesterol level. J. Lipid Res. 24:343-356.

52. Mak, K. M., and C. S. Lieber. 1984. Alterations in endothelial fenestrations in liver sinusoids of baboons fed alcohol. A scanning electron microscopic study. Hepatology. 4:386-391. 